\title{
SINUSOIDAL AND RELAXATION WIDE RANGE VOLTAGE CONTROLLED OSCILLATORS
}

This paper presents a sinusoidal Wien-bridge and square wave wide range voltage controlled oscillators. The square wave oscillator is based on relaxation (Schmitt trigger) topology. All described oscillators have potential use in different applications, e.g. phase locked loops, modulation and detection. The frequency changing is based on optically coupled resistor with $100 \mathrm{~dB}$ dynamic range. The oscillators were constructed and measured. The new method for amplitude control for quadrature oscillator is also described.

Keywords: Wien-bridge oscillator, relaxation oscillator, phase locked loop, voltage controlled oscillator, photoresistor, quadrature oscillator.

\section{Introduction}

A voltage controlled oscillator (VCO) is one of the important basic building blocks in analog and digital circuits. For example, a VCO is the main building block in phase locked loop (PLL) and clock generator circuits [1]. This paper presents the design of 2 types of oscillators that are continuously voltage tunable. The frequency changing for all oscillators is based on optically coupled photoresistor (Vactrol) with $100 \mathrm{~dB}$ dynamic range [2]. The first is Wien-bridge oscillator with frequency range from approx. $0.1 \mathrm{~Hz}$ to $250 \mathrm{kHz}$ with sinusoidal output. The Vactrol is used also for amplitude stabilization. The second oscillator is relaxation oscillator based on digital circuit with frequency range from $10 \mathrm{~Hz}$ to $9 \mathrm{MHz}$ with square wave output. It is important to note that the control voltage and oscillator part are optically coupled.

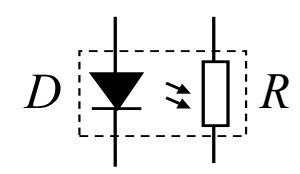

Fig. 1 The optically coupled photoresistor - Vactrol

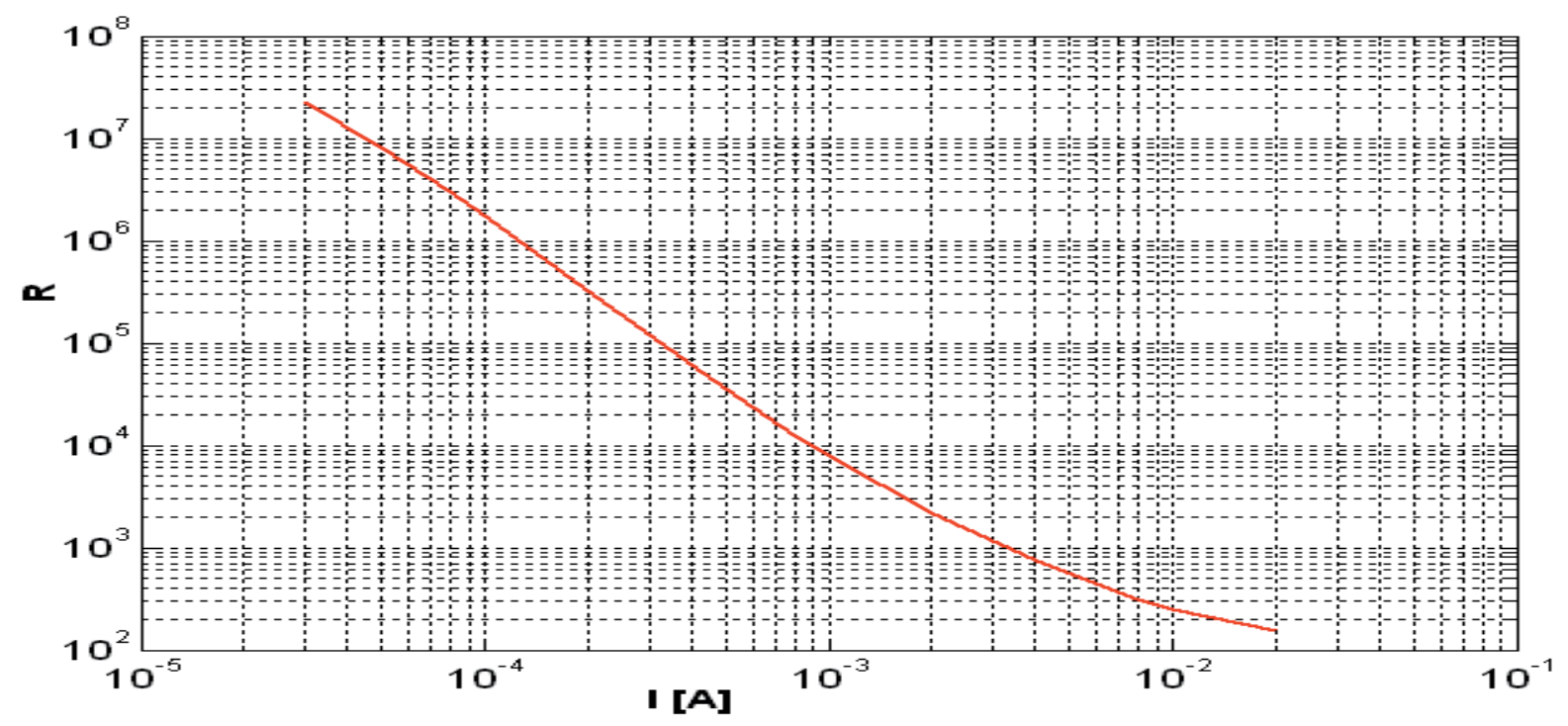

Fig. 2 Output resistance vs. input current for VTL5C1

\footnotetext{
* Milan Stork

Applied Electronics and Telecommunications/RICE, University of West Bohemia, Plzen, Czech Republic

E-mail: stork@kae.zcu.cz
} 


\section{Optically coupled photoresistor characteristic}

Vactrol consists of a LED diode and photoresistor (Fig. 1). A photoresistor or light-dependent resistor (LDR) or photocell is a resistor whose resistance decreases with increasing light intensity. Optically coupled photoresistor (OR), also called photoresistive opto-isolator or Vactrol (after a trademark introduced by Vactec, Inc.) offers $100 \mathrm{~dB}$ dynamic range, fast response time, and very high dark resistance. Some technical parameters for VTL5C1 [2] are: Min. isolation Voltage @ 70\% Rel. humidity: 2500 VRMS; Max. resistor power: $175 \mathrm{~mW}$; Max. resistor voltage: $100 \mathrm{~V}$; Max LED current: $40 \mathrm{~mA}$; Response time to $63 \%$ final $\mathrm{R}_{\mathrm{ON}} 2.5 \mathrm{~ms}$. The measured output resistance vs. input current is shown in Fig. 2 (logarithmic scales are used for both the $\mathrm{X}$ and $\mathrm{Y}$ axes).

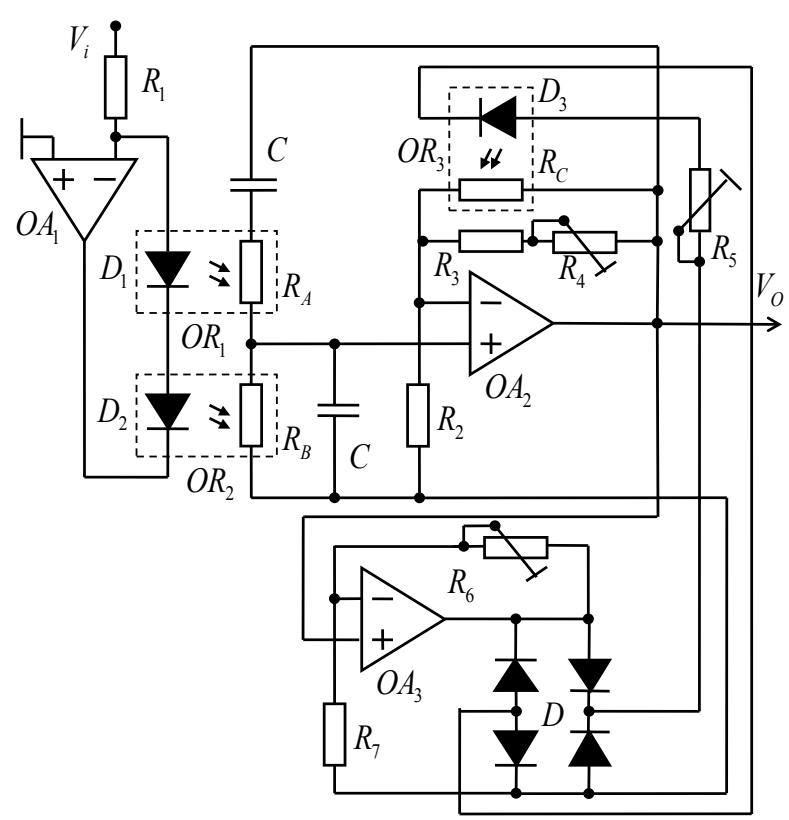

Fig. 3 The Voltage controlled Wien-bridge oscillator with buffer amplifier. $R_{1}=1 \mathrm{k} ; C=1 \mathrm{n} ; R_{2}=10 \mathrm{k} ; R_{3}=15 \mathrm{k} ; R_{4}=10 \mathrm{k} ; R_{5}=1 \mathrm{k} ; R_{6}=R_{7}=10 \mathrm{k}$;

OA-TL074; $D$ - 4x Schottky diodes, OR -OR optically coupled photoresistors VTL5C1.

\section{Wide range voltage controlled Wien-Bridge oscillator}

The voltage controlled Wien-bridge oscillator [3 - 5] is shown in Fig. 3. The operational amplifier $O A_{1}$ is used to form voltage controlled current source. The current $i_{\mathrm{D}}$, flows through diodes $D_{1}$ and $D_{2}$ of the $O R_{1}$ and $O R_{2}$. The output current is

$i_{D}=\frac{V_{i}}{R_{1}} \quad[\mathrm{~A}, \mathrm{~V}, \Omega]$

where $V_{\mathrm{i}}$ is input voltage and $R_{1}$ is resistor connected to inverting input.
The Wien-bridge oscillator consists of $O A_{2}$ where $C ; R_{\mathrm{A}} ; R_{\mathrm{B}}$ are in positive feedback. The buffer $O A_{3}$ and diode bridge $D, R_{3}$, $R_{4}, R_{5}$ and $O R_{3}$ are used for automatic gain control. The output frequency is given as

$$
f_{o}=\frac{1}{2 \pi C \sqrt{R_{A} R_{B}}} \quad[\mathrm{~Hz}, \mathrm{~F}, \Omega]
$$

Resistance of $O R$ is approximately

$$
R \approx \frac{k_{O R}}{i_{D}}=\frac{k_{O R}}{V_{i} / R_{1}}=\frac{k_{O R}}{V_{i}} \quad[\mathrm{~A}, \mathrm{~V}, \Omega]
$$

where $k_{\mathrm{OR}}$ is $O R$ constant and $i_{\mathrm{d}}$ is current through $O R$ LED diode. Suppose that the $R_{\mathrm{A}}=R_{\mathrm{B}}=R$ in this case the output frequency is given by

$f_{o}=\frac{1}{2 \pi C \sqrt{R_{A} R_{B}}}=\frac{1}{2 \pi C R} \approx \frac{V_{i}}{2 \pi C R_{1} k_{O R}}[\mathrm{~Hz}, \mathrm{~F}, \Omega]$

Measured output frequency vs. input voltage is shown in Fig. 4. The frequency spectrum is shown in Fig. 5 (spectral quality is better than $50 \mathrm{~dB}$ ).

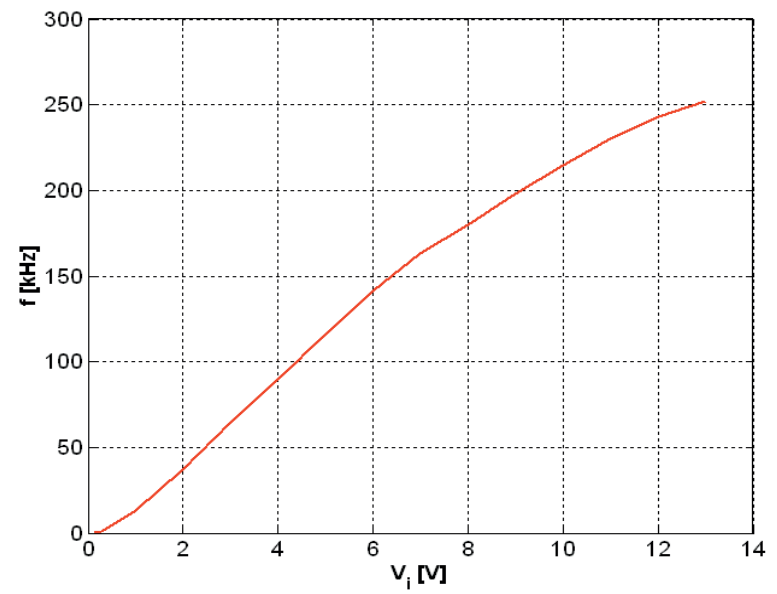

Fig. 4 Output frequency vs. input voltage for Wien-bridge oscillator

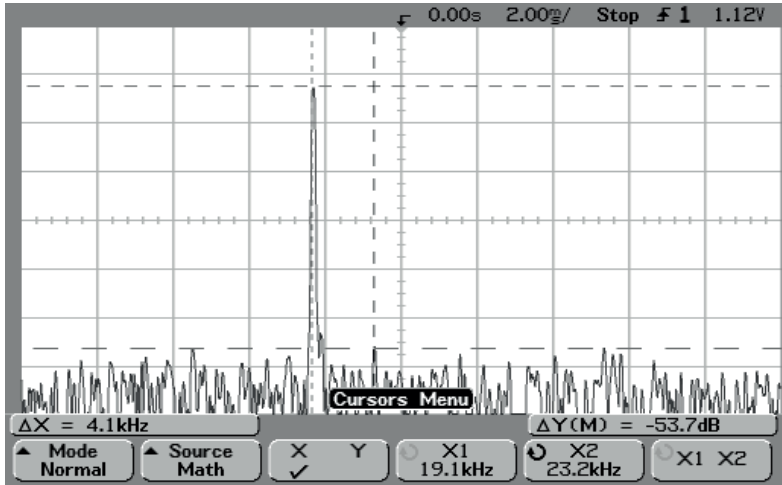

Fig. 5 The frequency spectrum of voltage controlled Wien-bridge oscillator 


\section{Amplitude control in quadrature sinusoidal oscillator}

In this part another principle of the amplitude control of the quadrature sinusoidal oscillator is used. The block diagram of the quadrature oscillator with amplitude (energy) stabilization is presented in Fig. 6. The method is based on compensation of the parasitic dissipation parameters $-\alpha_{1}$ and $-\alpha_{3}$ by the multipliers connected in parallel along the dissipation blocks [6 and 7]. Desired value of the amplitude $A$ of the oscillator signals is fed into the amplitude control block. The quadrature outputs of the oscillator $\left(x_{1}\right.$ and $\left.x_{2}\right)$ are also connected to the amplitude control block. Amplitude control is based on eq. (5) where ideal steady state is

$$
\begin{aligned}
& x_{1}^{2}+x_{2}^{2}=\left(V_{1} \sin (2 \pi f t)\right)^{2}+\left(V_{1} \cos (2 \pi f t)\right)^{2} \\
& =V_{1}^{2}(\underbrace{\sin (2 \pi f t)^{2}+\cos (2 \pi f t)^{2}})=V_{1}^{2}=A
\end{aligned}
$$

The amplitude is affected by means of PI (ProportionalIntegrated) controller and multipliers controlled by $x_{3}$.

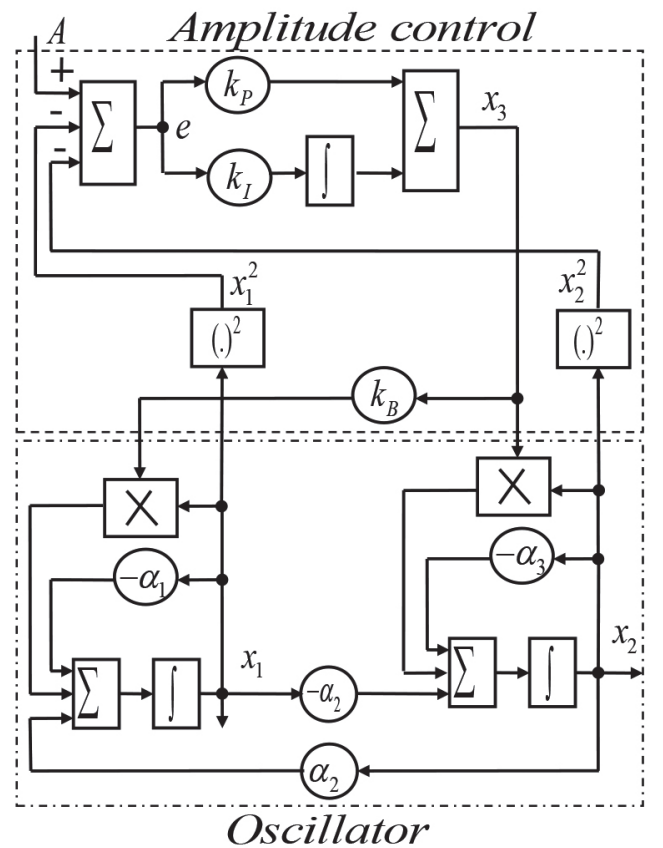

Fig. 6 Block diagram of the amplitude control for quadrature oscillator with compensation of dissipations $\alpha_{1}$ and $\alpha_{3}$. Controller for amplitude control is based on PI controller
$x_{3}=k_{p}\left(A-x_{1}^{2}-x_{2}^{2}\right)+k_{1} \int\left(A-x_{1}^{2}-x_{2}^{2}\right) d t$

and block $k_{\mathrm{B}}$ (in Fig. 6) is used for the balancing of different values of $\alpha_{1}$ and $\alpha_{3}$. In Fig. 7 the block diagram of compensated integrator is shown (top) and compensated integrator with OA (bottom). The compensated integrator with OA can be described by eq. (7) where $R_{4}\left(i_{d}\right)$ is photoresistor controlled by current $i_{d}$.

$\frac{V_{i}}{R_{i}}=-C \frac{d V_{O}}{d t}-\frac{V_{O}}{R_{D}}+\frac{R_{3} R_{4}\left(i_{d}\right) V_{O}}{R_{2}\left(R_{3}+R_{4}\left(i_{d}\right)\right) R_{5}}$

For ideal compensation of $R_{\mathrm{D}}$

$\frac{1}{R_{D}}-\frac{R_{3} R_{4}\left(i_{D}\right)}{R_{2} R_{5}\left(R_{3}+R_{4}\left(i_{D}\right)\right)}$

therefore

$R_{4}\left(i_{D}\right)=\frac{R_{2} R_{3} R_{5}}{R_{D} R_{3}-R_{2} R_{5}}$

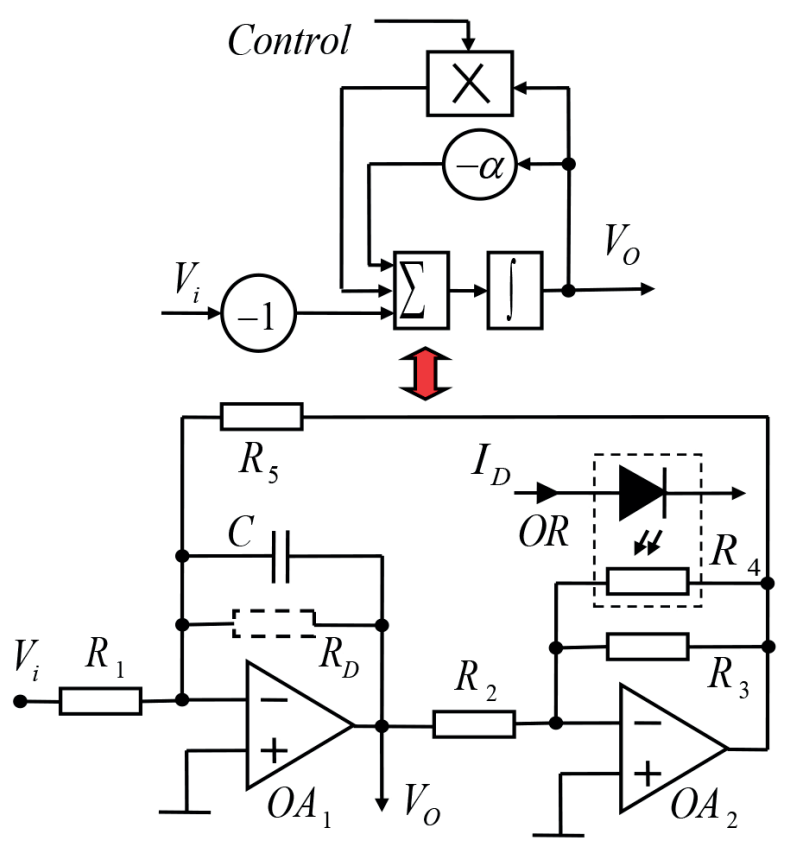

Fig. 7 The block diagram of amplitude control (top) and construction with optically coupled photoresistor (parasitic resistor $R_{D}$ is compensated) 


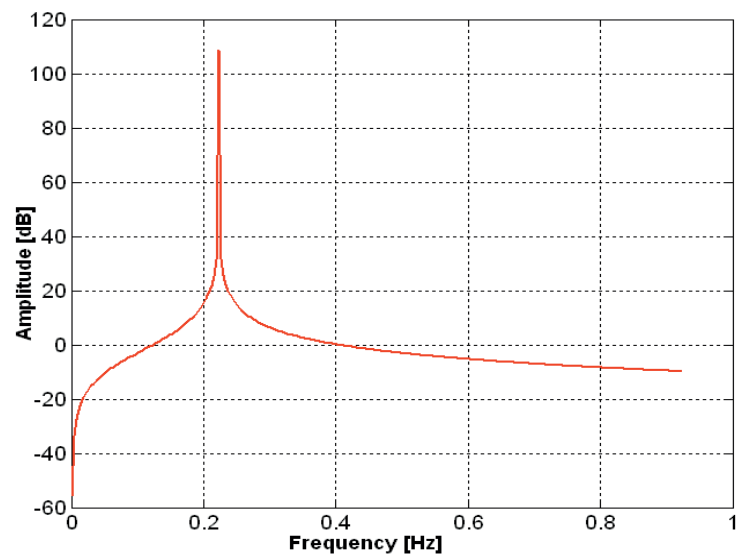

Fig. 8 Frequency spectrum of proposed oscillator (with compensation of the dissipation)

The frequency spectrum of quadrature oscillator with dissipative parameters compensation is shown in Fig. 8.

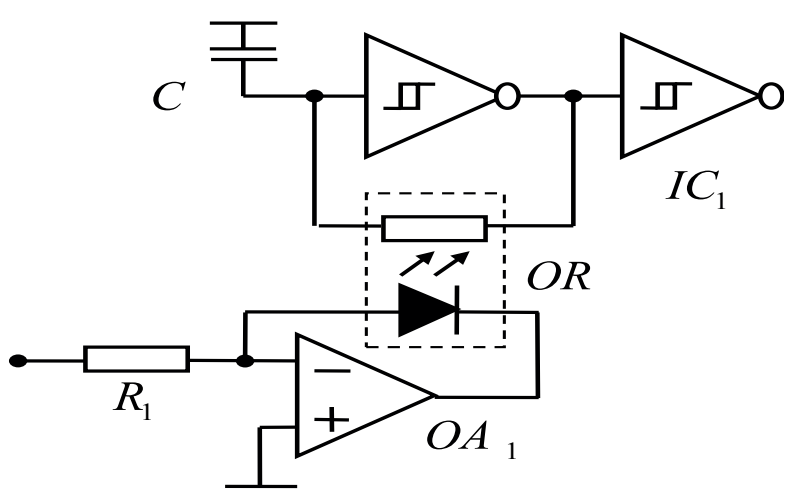

Fig. 9 The circuit diagram of relaxation oscillator with output buffer. $R_{I}=1 \mathrm{k} ; \mathrm{C}=56 \mathrm{pF}$; OA-TL071; IC - CD40109, OR - optically coupled photoresistors VTL5C1

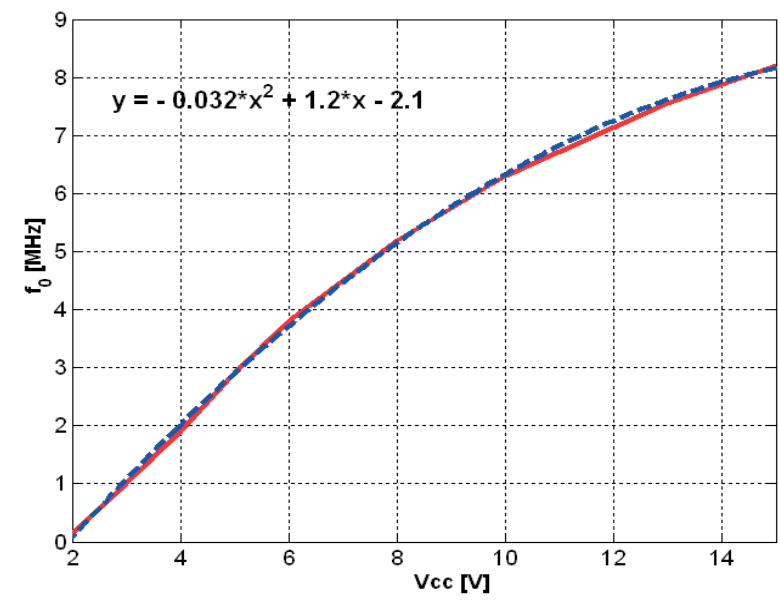

Fig. 10 Output frequency vs. supply voltage for relaxation oscillator with constant $V_{i}=13[\mathrm{~V}]$ (solid line), approximation - dash line

\section{Wide range voltage controlled relaxation oscillator}

The relaxation oscillator uses one Schmitt trigger capacitor and resistor [8 - 11]. The circuit diagram of voltage controlled relaxation oscillator with output buffer is shown in Fig. 9.

The output frequency vs. supply voltage (with fixed $V_{\mathrm{i}}=13$ [V]) is shown in Fig. 10. The output frequency vs. input voltage (with fixed $V_{\mathrm{CC}}=15[\mathrm{~V}]$ ) is displayed in Fig. 11.

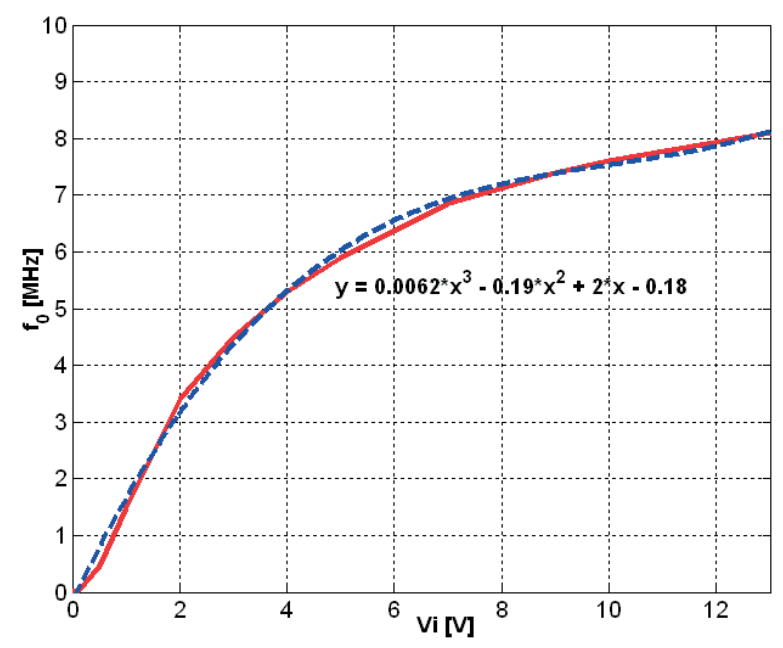

Fig. 11 Output frequency vs. input voltage for relaxation oscillator with $V c c=15$ [V] (solid line), approximation - dash line

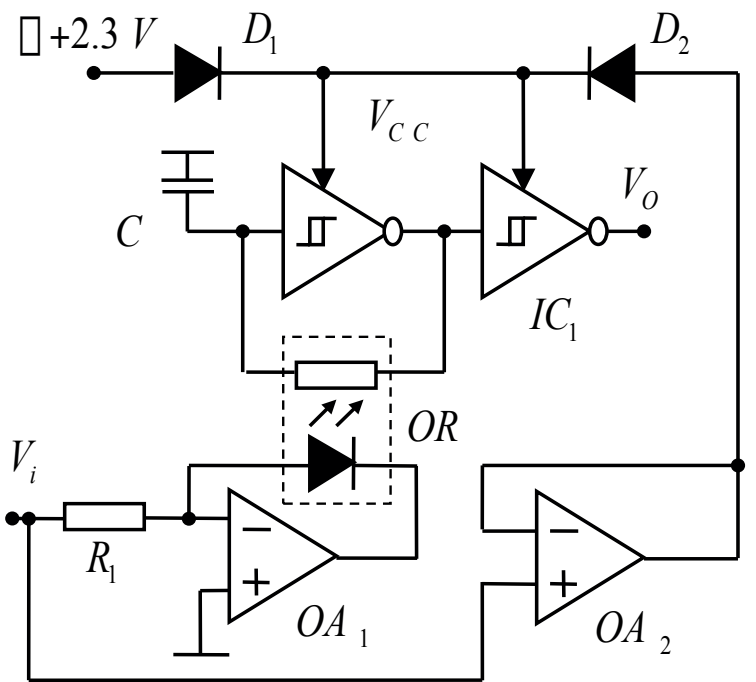

Fig. 12 The circuit diagram of linearized, relaxation oscillator with output buffer. $R_{1}=1 \mathrm{k} ; \mathrm{C}=56 \mathrm{pF} ; O \mathrm{~A}_{2}, O A_{2}-T L 072 ; I C_{1}-C D 40109, D_{P}, D_{2}$

- Schottky diodes, OR - optically coupled photoresistors VTL5CI 


\begin{tabular}{|c|c|c|c|c|c|c|c|c|c|}
\hline$V_{i}[\mathrm{~V}]$ & 2 & 3 & 4 & 5 & 6 & 7 & 8 & 9 & 10 \\
\hline$f_{0}[\mathrm{MHz}]$ & 0.12 & 0.9 & 1.7 & 2.6 & 3.5 & 4.3 & 5 & 5.8 & 6.4 \\
\hline
\end{tabular}

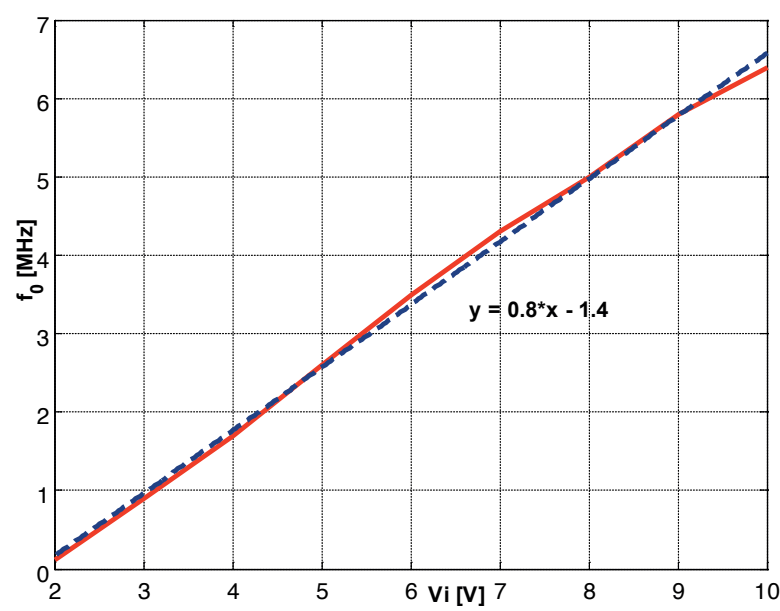

Fig. 13 Output frequency vs. input voltage for linearized relaxation oscillator (see Fig. 12). Measured values - solid line, approximation dash line

The circuit diagram of linearized relaxation oscillator with output buffer is shown in Fig. 13. Linearization is based on supply voltage increasing together with input voltage. The $\mathrm{OA}_{2}$ is used as power source for $V_{\mathrm{i}} \geq 2.3 \mathrm{~V}$. The supply voltage is given by eq. (10):

$V_{s S}= \begin{cases}\approx 2.3 & \text { if } V_{i}<2.3 \\ V_{i} & \text { if } V_{i} \geq 2.3\end{cases}$
Output frequency vs. input voltage of the linearized oscillator is displayed in Fig. 13. Measured values are in Table 1.

\section{Conclusion}

In this paper the 2 wide range simple V-f oscillators were described. For frequency control, the optically coupled photoresistor was used. The first is Wien-bridge oscillator with sinusoidal output with spectral quality greater than $50 \mathrm{~dB}$. The second is linearized relaxation oscillator. All oscillators were constructed and measured. It is important to note that these oscillators can be used in different applications including PLL, frequency locked loop and low cost frequency synthesizers. The new method for amplitude control of sinusoidal quadrature oscillators with high spectral quality based also on optically coupled photoresistors and PI controller was also described.

\section{Acknowledgment}

This research has been supported by the European Regional Development Fund and the Ministry of Education, Youth and Sports of the Czech Republic under the Regional Innovation Centre for Electrical Engineering (RICE), project No. CZ.1.05/2.1.00/03.0094 and by the Grant Agency of the West Bohemia in Pilsen, grant No. SGS-2015-002.

\section{References}

[1] POPENDA, A.: The DFM Control System Based on PLL, Communications - Scientific Letters of the University of Zilina, No.1, pp. 53-57, 2009.

[2] VTL5C1, 5C2, Low Cost Axial Vactrols, PerkinElmer Optoelectronics, www.perkinelmer.com/opto.

[3] LI, A.: Programmable Oscillator Uses Digital Potentiometers, Application note, AN-580, Analog Devices, 2002.

[4] MANCINI, R., PALMER, R.: Sine-Wave Oscillator, Application Report SLOA060 - March 2001, Texas Instruments, 2001.

[5] LINDBERG, E.: Oscillators - An Approach for a Better Understanding, Proc. of the 2003 European Conference on Circuit Theory and Design, Krakow, 2003.

[6] OLIVEIRA, L. B., FERNANDES, J. R., FILANOVSKY, I. M., VERHOEVEN, C. J. M., SILVA, M. M.: Analysis and Design of Quadrature Oscillators, ISBN: 978-1-4020-8515-4, Springer, 2008.

[7] JAIKIA, W., PROMMEE, P.: Electronically Tunable Current-mode Multiphase Sinusoidal Oscillator Employing CCCDTA-based Allpass Filters with Only Grounded Passive Elements, Radioengineering, vol. 20, No. 3, pp. 594-599, September 2011.

[8] LINSAY P.S., WANG, D. L.: Fast Numerical Integration of Relaxation Oscillator Networks Based on Singular Limit Solutions, IEEE Trans. Neural Net., 9: 523-532, 1998

[9] WEIGANDT, T. C, BEOMSUP, K., GRAY, P. R.: Analysis of Timing Jitter in CMOS Ring Oscillators, Proc. of IEEE Int. Symp. Circuits and Systems, vol. 4, pp. 27-30, London, June 1994.

[10] Accurate and Efficient Frequency Evaluation of a Ring Oscillator, Application Note 4070-3, Agilent Technologies 2000. 
[11] YUCHI, NI: Low-power CMOS Relaxation Oscillator Design with an On-chip Circuit for Combined Temperature-compensated Reference Voltage and Current Generation, Northeastern University, Electrical and Computer Engineering Master's Theses. Paper 127, 2014 http://hdl.handle.net/2047/d20004909. 